\title{
Technical Note : Development of a 3D Printed Subresolution Sandwich Phantom for Validation of Brain SPECT Analysis
}

lan S Negus ${ }^{\mathrm{a}}$

Dr Robin B Holmes ${ }^{\mathrm{a}}$

5 Kirsty C Jordan ${ }^{\text {b }}$

David A Nash ${ }^{\mathrm{C}}$

Dr Gareth C Thorne ${ }^{a}$

Dr Margaret Saunders ${ }^{a}$

a Department of Medical Physics and Bioengineering, University Hospitals Bristol NHS

10 Foundation Trust, United Kingdom

${ }^{\mathrm{b}}$ Department of Biomedical Engineering, University of Strathclyde, United Kingdom

${ }^{c}$ Department of Medical Physics, Portsmouth Hospitals NHS Trust, United Kingdom

\section{Keywords}

3D Printing, nuclear medicine, phantoms, quantitative imaging, brain 


\section{Abstract \\ Purpose}

To make an adaptable, head shaped radionuclide phantom to simulate molecular imaging of the brain using clinical acquisition and reconstruction protocols. This will allow the characterisation and correction of scanner characteristics, and improve the accuracy of clinical image analysis, including the application of databases of normal subjects.

\section{Methods:}

A fused deposition modelling 3D printer was used to create a head shaped phantom made up of transaxial slabs, derived from a simulated MRI dataset. The attenuation of the printed polylactide (PLA), measured by means of the Hounsfield unit on CT scanning, was set to match that of the brain by adjusting the proportion of plastic filament and air (fill ratio). Transmission measurements were made to verify the attenuation of the printed slabs. The radionuclide distribution within the phantom was created by adding ${ }^{99 \mathrm{~m} T c}$ pertechnetate to the ink cartridge of a paper printer and printing images of gray and white matter anatomy, segmented from the same MRI data. The complete subresolution sandwich phantom was assembled from alternate 3D printed slabs and radioactive paper sheets, and then imaged on a dual headed gamma camera to simulate an HMPAO SPECT scan.

\section{Results:}

Reconstructions of phantom scans successfully used automated ellipse fitting to apply attenuation correction. This removed the variability inherent in manual application of attenuation correction and registration inherent in existing cylindrical phantom designs. The resulting images were assessed visually and by count profiles and found to be similar to those from an existing elliptical PMMA phantom.

\section{Conclusions:}


We have demonstrated the ability to create physically realistic HMPAO SPECT simulations using a novel head-shaped 3D printed subresolution sandwich method phantom. The phantom can be used to validate all neurological SPECT imaging 45 applications. A simple modification of the phantom design to use thinner slabs would make it suitable for use in PET. 


\section{Introduction}

Analysis of regional cerebral blood flow (rCBF) single photon emission computed tomography (SPECT) is an important part of the assessment of patients with suspected dementia ${ }^{1}$. This may be interpreted visually from a series of planar images through the brain, but this process is relatively insensitive to subtle blood flow loss and is subject to observer variability ${ }^{2}$. Computer analysis that fits the image dataset to a standard geometry and compares it to a 'normal database' has been used clinically and in research. These age matched databases are taken from volunteer controls or a representative normal patient cohort ${ }^{3-5}$. Several software packages are available that allow automated whole-brain analysis of brain SPECT studies ${ }^{3,6-8}$.

A limitation of SPECT is that different gamma cameras and collimators will yield slightly different image data for a given distribution of activity. In rCBF SPECT this will both affect the visual appearance and limit the applicability of normal databases to images acquired on different cameras. In order to characterize, and if necessary correct for, these variations, a physical phantom can be used that mimics both the radionuclide activity distribution and the photon attenuation in a patient ${ }^{9-12}$. An anthropomorphic phantom will allow clinically realistic testing of image reconstruction, including attenuation and scatter correction and resolution recovery.

The Hoffman fillable phantom is commercially available and used to model the activity of gray and white matter ${ }^{13}$. However, this has a fixed geometry that, with most versions, does not include the whole brain. This prevents count normalization to the cerebellum and makes lesion simulation difficult. In addition the fixed gray and white matter volumes, and hence relative activity, are not suitable for simulating all scan types. This includes HMPAO SPECT studies of normal function, where the Hoffman phantom's 4:1 gray to white matter ratio does not deliver the required ratio of approximately $2: 1^{14}$. 
The subresolution sandwich method (SSM) is derived from the stack phantom design of Larsson et al. ${ }^{15}$ and has been used in previous studies to simulate radionuclide scans of the human brain using SPECT and PET 8, 16, 17. An SSM phantom is an assembly of individual sheets of paper, separated by slabs of tissue equivalent material, at a distance of less than half the resolution of the imaging device. Radioactive images are printed on the paper sheets using a radioactive solution to build a 3D activity distribution. This method can simulate highly realistic and easily adaptable images for both SPECT and PET.

The construction of existing phantoms from equal sized circular or elliptical sheets of poly (methyl methacrylate) (PMMA) has limitations ${ }^{8,16,17}$. The non-anthropomorphic shape is not physically realistic in terms of gamma ray attenuation and scatter. Additionally, automated uniform attenuation correction cannot be used, because the edge of the activity is not the edge of the attenuation. A method was therefore required to produce head shaped tissue equivalent sheets. 3D printing, or additive manufacturing, technology has been proven able to produce complex distributions of polymers similar to PMMA from DICOM datasets from CT or MRI ${ }^{18}$.

A number of additive manufacturing approaches have been used to create anthropomorphic phantoms for radionuclide imaging. Fillable phantoms have been produced that overcome many of the limitations of the Hoffman phantom ${ }^{19}$ or offer patient specific organ geometry ${ }^{20}$. For PET, a powder deposition printer, with $18 \mathrm{~F}$ mixed into the binding resin has been used to create a brain phantom ${ }^{21}$. However, there are disadvantages to these approaches. Resin phantoms with clinical radionuclides have a very short useful life, while images of liquid filled phantoms may be affected by cold boundaries 22, 23. In contrast to SSM phantoms, for replicating clinical radionuclide 
distributions, these approaches are restricted to producing volumes of fixed and largely uniform activity.

Applying 3D printing to the manufacture of a SSM phantom requires a material that replicates the radiation attenuation of the head at the emission energy of the radionuclide used. The National Institute of Standards and Technology gives a mass attenuation coefficient of 0.17 and $0.15 \mathrm{~cm}^{2} / \mathrm{g}$ for ICRU brain at 100 and $150 \mathrm{keV}$ respectively ${ }^{24}$. Interpolating and correcting for a density of $1.04 \mathrm{~g} / \mathrm{cm}^{3}$ gives an attenuation coefficient of $0.16 \mathrm{~cm}^{-1}$ at $140 \mathrm{keV}\left({ }^{99 \mathrm{mT}} \mathrm{T}\right)$. lida et al. measured the attenuation for stereo lithography photopolymer as $0.161 \pm 0.006 \mathrm{~cm}^{-1}$ at $140 \mathrm{keV}^{19}$. They used a $\mathrm{K}_{2} \mathrm{HPO}_{4}$ solution for the skull. More generally, a range of additive manufacturing materials have been characterized using CT, showing materials approximating fat, soft tissue and bone 25,26 . Printers using curable liquid photopolymers have been used to make phantoms representing breast, lung and soft tissue masses ${ }^{26-28}$. Tuning of radiation attenuation properties has been achieved by doping the binder of a powder deposition printer with $\mathrm{Nal}^{29}$ and by adjusting the proportions of polymer and air (fill density) in fused deposition modelling (FDM) ${ }^{30}$. 


\section{Materials and Methods}

115 A RepRapPro Mendel 3D printer (RepRapPro, Bristol, UK) was purchased in kit form, assembled and calibrated. This was a commercially available example of the open source RepRap printer, developed at the University of Bath, UK ${ }^{31}$. It had one extruder enabling it to print in a single material. The printer was operated using Pronterface open source software ${ }^{32}$. Individual print (G-code) files were created with Slic3r software 120 (version 0.9$)^{33}$, with a constant fill density set for each print. All 3D printer feedstock, as $1.75 \mathrm{~mm}$ diameter PLA filament, was manufactured and supplied by Faberdashery (Frome, UK).

The variation of attenuation with fill density was investigated by printing a series of $2 \mathrm{~cm}$ cubes at different fill density settings (set in Slic3r). These were scanned on a Siemens

125 Sensation 16 scanner (Siemens Healthcare, Erlangen, Germany) with a standard axial head protocol ( $\mathrm{H} 31$ algorithm, $3 \mathrm{~mm}$ reconstructed slice width). A single region of interest was positioned on each image, central to the cube, avoiding the edges, and the Hounsfield Unit (HU) value and standard deviation recorded. A linear relationship was seen up to a nominal fill density of $95 \%(154 \mathrm{HU})$, with a maximum $\mathrm{HU}$ of 164 , as shown in figure 1.

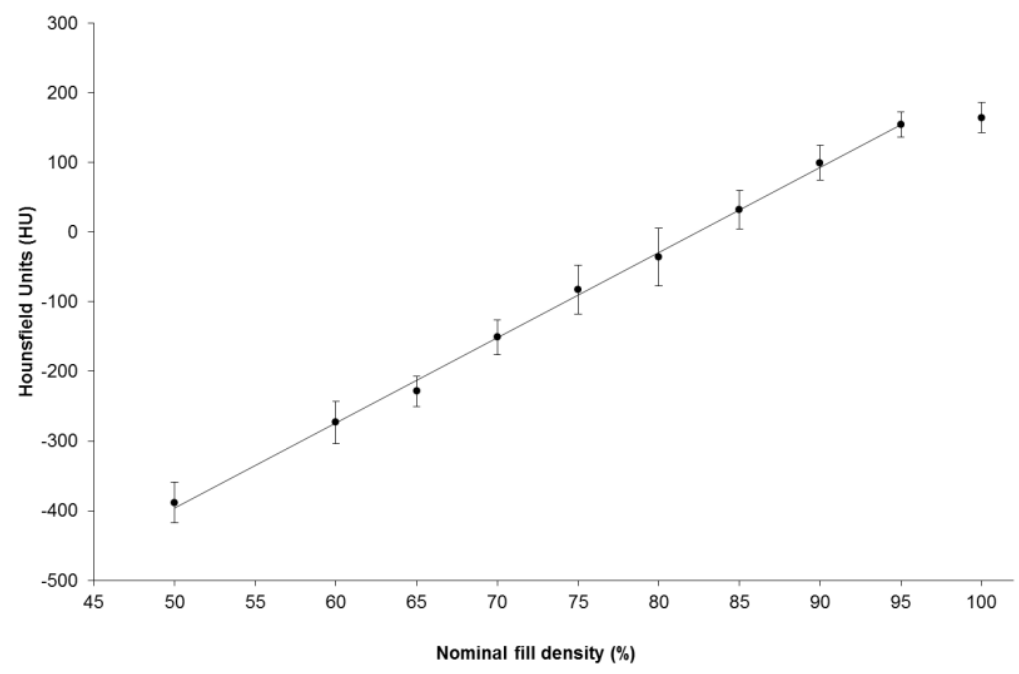


Figure 1 Measured variation of CT Hounsfield Unit with print fill density

The fact that the response is not linear up to a nominal $100 \%$ appears to be due, at least in part, to the extruder feed rate setting, which will be specific to the printer. Excluding the value at $100 \%$ fill density, the following regression equation was calculated at $120 \mathrm{kVp}$ : $H U=(12.2 \pm 0.5) *(\%$ fill density $)-(1008 \pm 35)$

No notable difference in this relationship was seen on a Philips Big Bore CT scanner (Philips Medical Systems, Best, NL) at 90, 120 or $140 \mathrm{kVp}$. A fill density of $85 \%$ was chosen to give $30 \mathrm{HU}$, the value for brain being in the approximate range $20-40 \mathrm{HU}^{34}$.

140 The head volume data to manufacture the phantom was taken from the Brainweb online database of simulated MRI scans ${ }^{35-38}$. Subject number 4 was used, which consists of a head volume including the whole brain but excluding the mouth. A number of software packages were then used to isolate the head volume, convert this to the required stereolithography (STL) format and divide the volume into slabs. All tissue voxel values were set to equal one in MATLAB (MathWorks, Natick, MA). The outline of the volume was then determined in ITK-snap, by automatically segmenting the head volume from the background ${ }^{39}$. The resultant NIfTI file was converted to STL format by creating a grayscale model of the head volume in 3D Slicer, setting the voxel value threshold to 0.5 40. The slabs, of thickness $4 \mathrm{~mm}$, were then created in netfabb (netfabb, Lupburg, 150 Germany). Finally Slic3r was used to create the printer instruction G-code for each slab. Print layer height was $0.4 \mathrm{~mm}$, except for the first which was $0.3 \mathrm{~mm}$ to improve adhesion to the printer bed and reduce overall height to allow for the paper. Each slab of the phantom was printed, in turn, and labelled.

The narrow beam attenuation coefficient of the printed slabs was estimated by measuring the transmission with a ${ }^{99 m}$ Tc source. An Unfors Raysafe (Billdal, Sweden) Xi survey ionisation chamber, of diameter $6 \mathrm{~cm}$, was positioned $50 \mathrm{~cm}$ above a vial 
containing $4 \mathrm{GBq}{ }^{99 \mathrm{~m}} \mathrm{Tc}$ pertechnetate in $4 \mathrm{ml}$ solution. The vial was positioned in an 18 $\mathrm{mm}$ thick lead pot with a $10 \mathrm{~mm}$ diameter hole drilled centrally in the lid. The divergent beam of photons covered the detector area. The dose rate at the detector was recorded, and then the $4 \mathrm{~mm}$ slabs were placed incrementally on top of the lead pot, with the dose rate recorded each time, until a transmission of $40 \%$ was measured.

Brain activity distributions were derived from the same simulated MRI scan used to create the phantom. Grayscale values for gray and white matter were chosen to give a GM:WM ratio of 2:1, which was validated by counting printed paper samples in a gamma counter. The resultant images represented $2 \times 2 \times 4 \mathrm{~mm}$ voxels suitable for use in the phantom. An aqueous solution of $4 \mathrm{GBq}{ }^{99 \mathrm{~m} T c}$ pertechnetate in $4 \mathrm{ml}$ volume was added to the part full ink cartridge of an HP Officejet Pro 8100 printer (Hewlett Packard, Palo Alto, California) and the images were printed.

The phantom was assembled and held rigid by means of two $5 \mathrm{~mm}$ threaded nylon bars running vertically through holes in each slab. The location of the holes was decided by examining the brain structure to best avoid areas of activity, passing through the ventricles where possible. Corresponding holes were drilled through the stack of printed paper sheets prior to assembly. After assembly, excess paper was cut from the assembled phantom to ensure this did not affect the positioning in the gamma camera.

175 Filling the printer cartridge, printing the 41 images and assembling the phantom took approximately an hour. A second set of images was printed, on the same day and using the same printer, and assembled into the elliptical PMMA phantom described by Berthon 17. Each phantom was imaged on a GE DST XLi dual-headed gamma camera (GE Healthcare, Little Chalfont, UK) with low-energy high resolution parallel hole collimators. Before acquisition, the phantom was positioned in the patient headrest, with the slabs 
and printed sheets orthogonal to the axis of rotation, using a spirit level to align in the sagittal and axial planes. Alignment in the coronal plane was judged by eye in the absence of a laser. Imaging consisted of 128 projections over 360 degrees, using a zoom of 1.33 to give a pixel size of $3.38 \mathrm{~mm}$. The time per projection was calculated from a static measure of count rate to give a total of 5 million counts for the acquisition. Reconstruction with attenuation correction was carried out in two stages. An initial filtered back-projection reconstruction was used to fit an ellipse to the edge of the brain on a slice-by-slice basis. For the 3D printed head shaped phantom, this process was automated, as is the clinical routine for this institution. For the elliptical PMMA phantom, the size of the ellipse was set manually using GE Vision software by visual identification of the partial ring of activity from a printed paper sheet wrapped around the back of the phantom. The resulting set of ellipses for each phantom was used to apply a uniform attenuation correction in the second reconstruction. Typically used attenuation coefficients for water at $140 \mathrm{keV}$ are $0.15 \mathrm{~cm}^{-1}$ (narrow beam) and $0.12 \mathrm{~cm}^{-1}$ (broad beam $)^{41}$. However, $0.112 \mathrm{~cm}^{-1}$ (broad beam) had previously been shown to give optimal image uniformity for uniform activity phantom measurements on this camera ${ }^{42}$. A nominal broad beam attenuation coefficient of $0.112 \mathrm{~cm}^{-1}$ was set for both phantoms.

The second filtered back-projection for each phantom used a ramp filter on $128 \times 128$ matrices, with an isotropic voxel size of $3.38 \mathrm{~mm}$. The spatial resolution (full width half maximum) was previously demonstrated to be $10 \mathrm{~mm}$ on reconstructed cuts ${ }^{16}$. A $10 \mathrm{~mm}$ smoothing filter was applied to reduce visible noise.

For image presentation, each set of images was registered to the source (paper printing template) data in SPM8 ${ }^{43}$, and counts globally scaled in MATLAB to give a mean count for the brain of 100 . This gave axial, coronal and (para)sagittal views, along the same planes, demonstrated in the results. For comparison, the source data was corrected for 
the printer grayscale vs. activity response, smoothed, and presented in the same format. Count profiles were taken for each of the 3DP phantom, elliptical phantom and source data images, along two lines left to right through the front and rear of a mid brain axial 210 slice. 


\section{Results}

The narrow beam attenuation coefficient of the printed slabs was taken from an exponential fit of 11 transmission measurements plotted against cumulative thickness in Microsoft Excel, and found to be $0.167 \mathrm{~cm}^{-1}\left(\mathrm{R}^{2}=0.997\right)$. The mean thickness of the 215 slabs, measured from an assembled stack of 20 , was $3.90 \pm 0.05 \mathrm{~mm}$. The mean thickness of a sheet of A4 office paper ( $80 \mathrm{gsm}$ ), again measured from a stack of 20 sheets, was $0.10 \mathrm{~mm}$, giving an assembled slice thickness of the slabs and paper of 4 $\mathrm{mm}$. The attenuation coefficient of paper was not assessed but has a nominal density of $0.8 \mathrm{gcm}^{-3}$, indicating a lower attenuation than soft tissue. The mean thickness of 220 individual slabs was $3.78 \pm 0.15 \mathrm{~mm}$, indicating that imperfections in the top surface are leading to air gaps of approximately $0.1 \mathrm{~mm}$. Using the measured slab thicknesses gave $0.172 \mathrm{~cm}^{-1}$ for the printed material $\left(R^{2}=0.998\right)$.

The assembled subresolution sandwich (SSM) phantom is shown in figure 2.

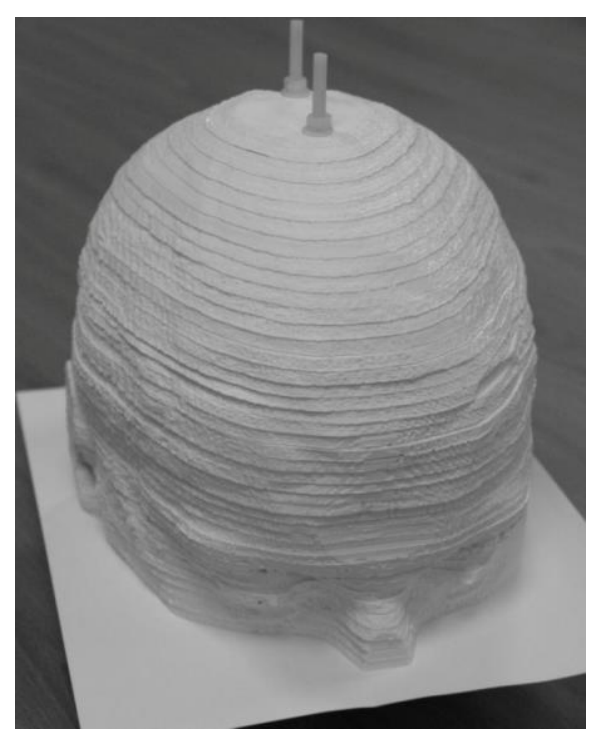

Figure 2 The assembled SSM phantom. A single sheet of paper is shown. When assembled for imaging, printed brain activity distributions on paper are placed between each slab. 
This illustrates the $3.9 \mathrm{~mm}$ thick PLA slabs and the threaded nylon bars that hold it together. Fully assembled, the phantom has a sheet of paper, with the corresponding anatomical activity distribution printed in radioactive ink, sandwiched between each slab. For clarity, a single sheet of paper is shown between the two most caudal slabs to demonstrate the SSM principle. Figure 3 shows the grayscale image of activity printed on paper in a midbrain axial slice.

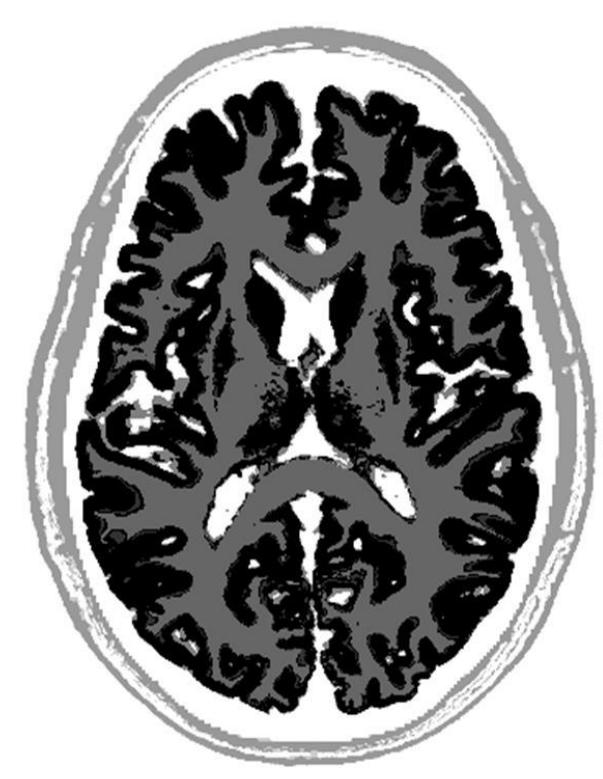

Figure 3 An example grayscale image for printing on paper

Gray matter appears black, and was calibrated to have twice the activity density as the white matter, which appears gray in the image. Printed activity outside the brain approximates uptake in areas including the scalp.

The assembled 3D printed anthropomorphic phantom was imaged and example reconstructed images are shown in figure 4. 


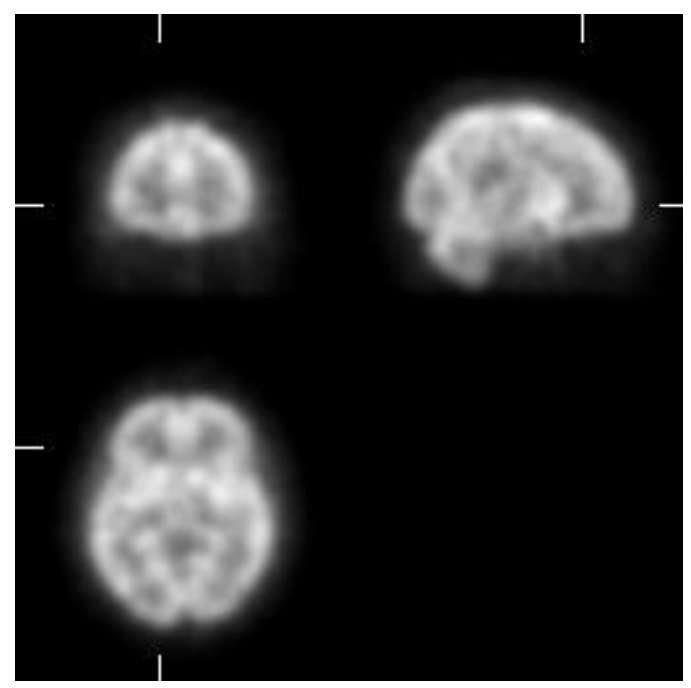

Figure 4 Coronal, sagittal and axial reconstructed slices from the anthropomorphic 3D printed phantom

Coronal, sagittal and axial planes are shown, clockwise from top left. The positions of the displayed orthogonal planes are marked, and are clearly shown on the source data images in figure 6.

As described above, the same paper printed distributions were assembled into an elliptical PMMA phantom. Figure 5 shows the reconstructed coronal, sagittal and axial images from the elliptical phantom.

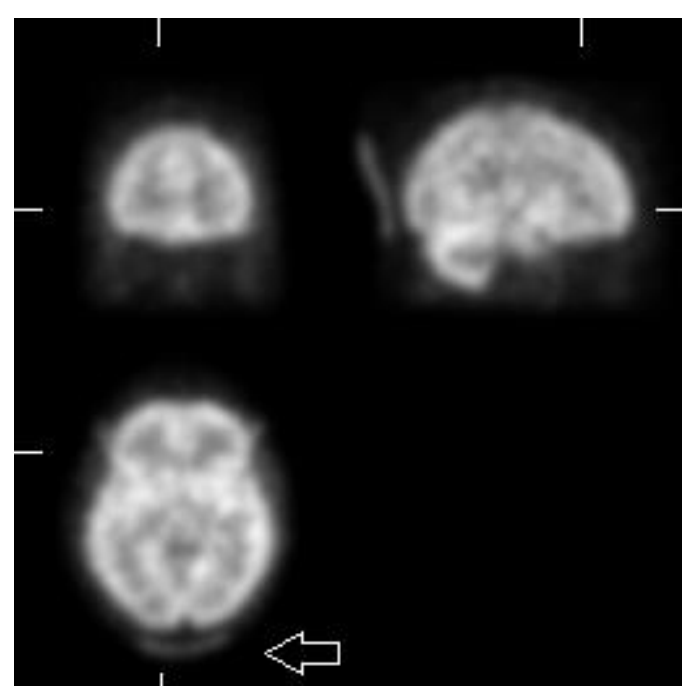


Figure 5 Coronal, sagittal and axial reconstructed slices from the elliptical PMMA phantom. The arrow shows the position of the additional activity to delineate the edge of the phantom

The image planes are at the same positions. Figure 5 also shows, arrowed, an additional sheet of paper, with radioactive ink, taped to the back of the elliptical phantom, required to delineate the phantom outline when manually applying attenuation correction. The sagittal image shows that this sheet of paper distorted when the phantom was positioned, which is a potential source of error in the technique. For comparison, the source data used to create the radioactive ink distribution is presented in the same format in figure 6.

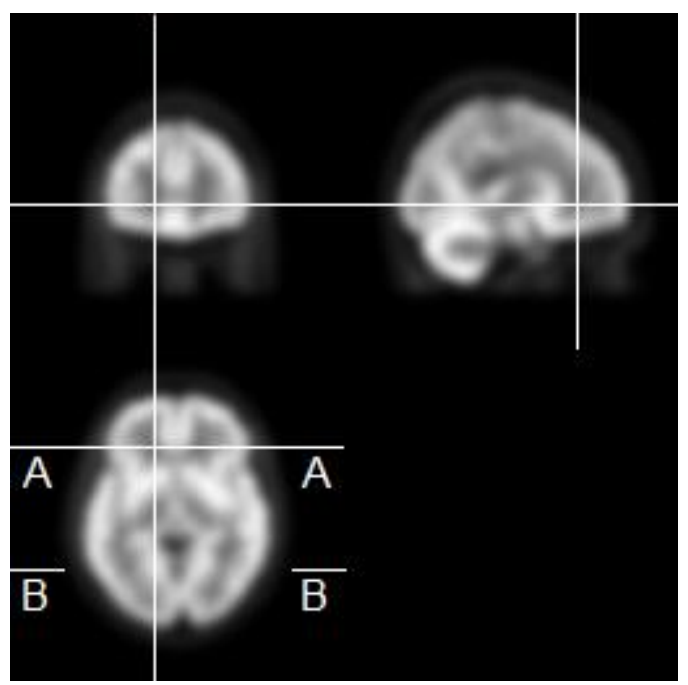

Figure 6 Coronal, sagittal and axial slices from the smoothed source data. The lines $A-A$ and $B-B$ show the position of the count profiles through the axial slice. This shows, as continuous lines, the positions of the displayed image planes. Also shown are the positions of the count profiles, taken along the lines $A-A$ and $B-B$ through the displayed axial slice for each phantom and the source data.

Figure 7 shows count profiles for the 3D printed phantom, elliptical phantom and source data along the line A-A, left to right across the front of the brain. 


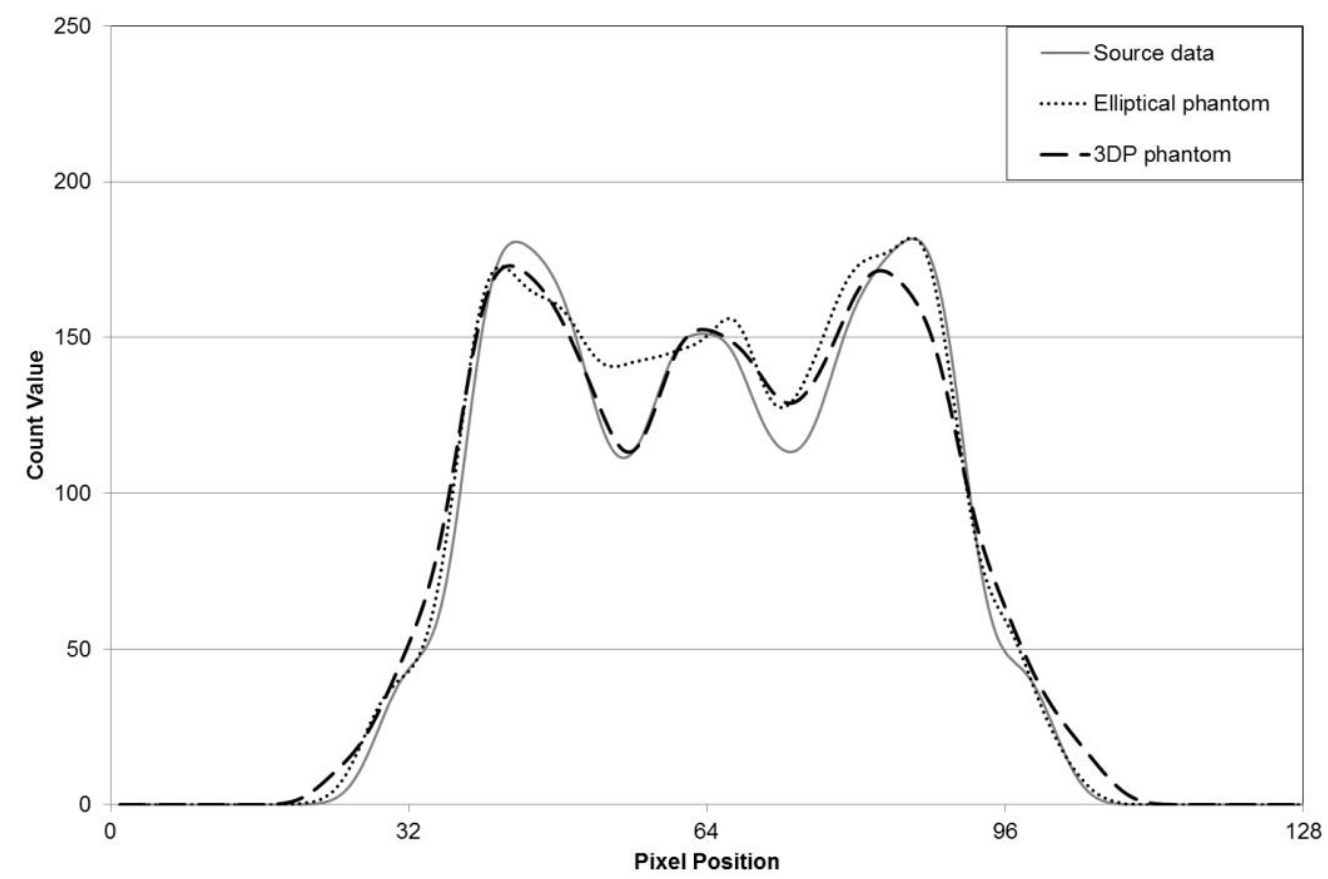

Figure 7 Count profiles along the line A-A

The count profiles along the corresponding line B-B, left to right across the rear of the brain are shown in figure 8.

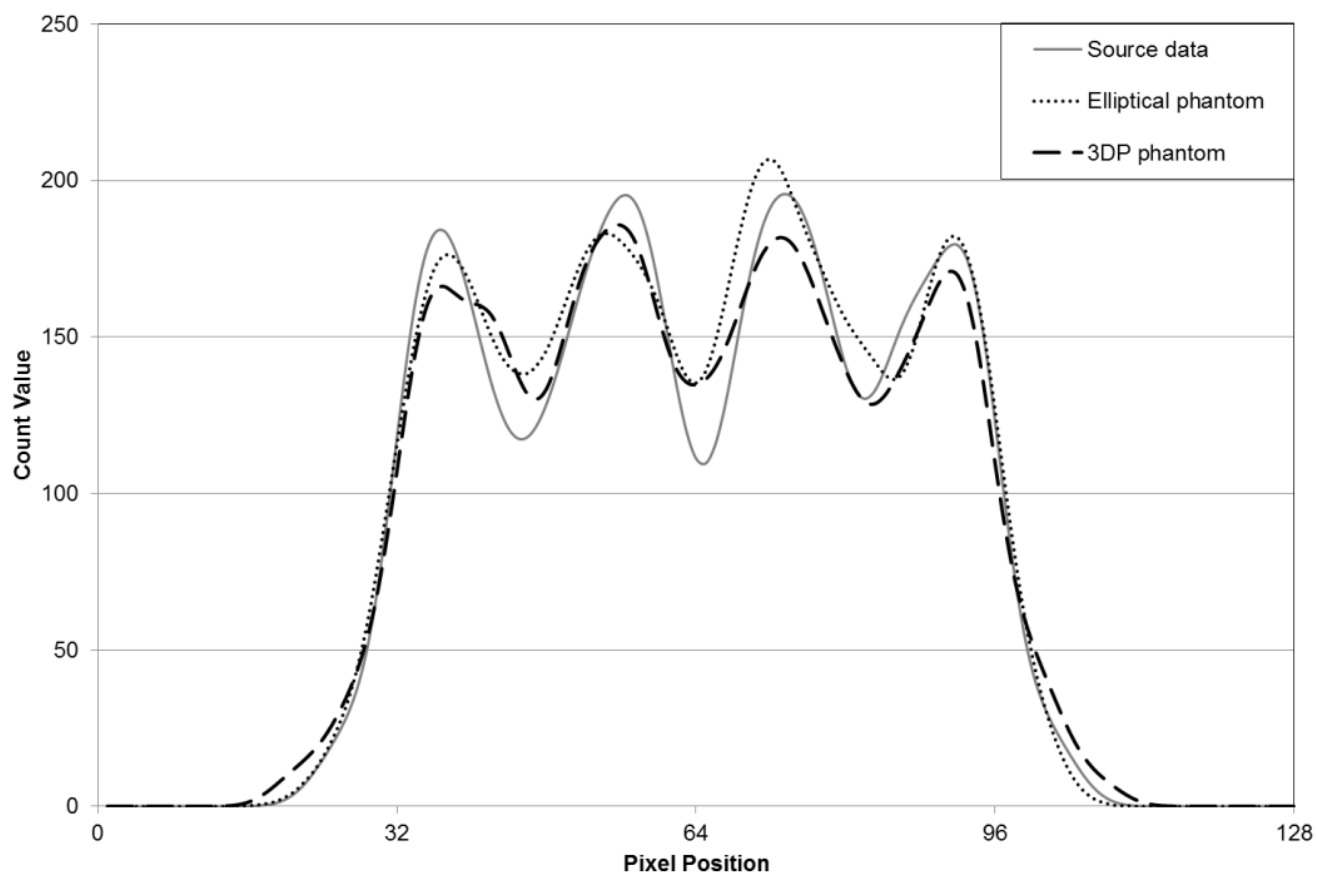

Figure 8 Count profiles along the line B-B 


\section{Discussion}

A head shaped SSM phantom was constructed in a tissue equivalent material using a fused deposition modelling (FDM) 3D printer. Reconstructions of the phantom scans successfully used automated ellipse fitting to apply attenuation correction. This removes the variability inherent in manual application of attenuation correction and registration inherent in existing cylindrical phantom designs. Count profiles through the resulting images showed a similar resolution of areas of low and high activity to an existing elliptical PMMA phantom ${ }^{17}$. While figure 7 appears to show better differentiation of a low activity area to the left, no such difference is seen in figure 8. The anthropomorphic phantom will give more realistic absorption and scatter than an elliptical cylinder, but images with much higher counts would be required to adequately determine the difference between the phantoms. The demonstrated advantage of the phantom on a gamma camera without measured attenuation or scatter correction, is that clinical reconstruction and image processing can be applied to all stages in the phantom simulation.

Many SPECT and PET scanners apply more advanced corrections for attenuation (often utilising CT data), scatter and resolution. Characterising these scanners requires phantoms that reflect the geometry of the patient. Joshi et al. showed that the cylindrical Hoffman phantom did not adequately correct for inter-scanner differences in FDG PET imaging ${ }^{44}$. This was in part due to the cylindrical shape, and the lack of both a skull and scattering material in the neck. Secondly, corrections derived from a single phantom may not apply to the range of patient's heads size. The need for a realistic anthropomorphic phantom to correct for inter-scanner differences is likely to be more pronounced for SPECT, due to differences in physical collimation, but this has not yet been assessed. 
The measured narrow beam attenuation coefficient of the slabs in an assembled stack was $0.167 \mathrm{~cm}^{-1}$, which is very similar to the clinical whole head attenuation measurements by lida at $0.166 \mathrm{~cm}^{-1} 45$, but slightly higher than that of brain at 0.16 $\mathrm{cm}^{-124}$. This is satisfactory, considering that CT was used to predict the required print density, but there are several sources of error in the estimate. Attenuation measurements did not include the paper sheets which account for $2.5 \%$ of the thickness of the assembled phantom and have a lower density than the PLA slabs. Non uniformity in attenuation will have been caused by the thinner (and denser) first print layer, and by the observed imperfections in the slab top surface; the bottom of each slab, having been melted onto the glass printer bed, is flat but the top could be sanded smooth. An additional threaded rod may be required to keep the phantom rigid, as the friction between layers would be lower. Measurements of HU by CT showed attenuation varies linearly with print density so the attenuation, based on measurements with paper in situ, could be adjusted in subsequent prints.

The use of the print fill density to introduce air between the extruded threads of polymer and adjust the attenuation properties is suitable for imaging by SPECT or PET. The resolution for both modalities is much less than the scale of the extruded threads. A modified phantom for PET would ideally tune the material attenuation to match the head at $511 \mathrm{keV}$, with slabs of approximately $2 \mathrm{~mm}$ thickness. However, for FDM to be realistic at the sub $\mathrm{mm}$ scale, e.g. for CT, it is envisaged that modified or novel printer feedstock materials would be required, printed at $100 \%$ fill.

Further realism in the attenuation properties of the phantom could be achieved by adjusting the $3 \mathrm{D}$ printed material distribution. The software (Slic3r) that we used to produce the printer instructions (G-code) does not allow for continuously varying fill density, but there are two potential methods to create areas of different density. One 
approach is use the facility in Slic3r to superimpose an additional object (STL file) as a 'modifier mesh' with a different fill density. Alternatively up to three objects can be combined into one print, with a different extruder printing each volume. This gives the potential for adjusting flow rate and/or using different feedstock materials. The realism of the phantom could be improved by modelling the skull in a higher attenuation material ${ }^{44,46}$ and future work will look to develop this.

A disadvantage of the FDM technique is that it is relatively time consuming; each of the 42 slabs took 3 to 4 hours to print, with approximately 30 minutes required for cooling. While a small reduction in print time may be possible by optimising settings, it was found that a faster extrusion rate can give uneven prints. This restriction is inherent to the FDM printing method. The use of a commercial rather than kit built FDM printer could save time in calibration and adjustment, and better ensure reliable unattended operation, at the cost of limiting options for multiple extruders with different materials.

Functional brain imaging using PET and SPECT is widely used in the diagnosis and characterisation of a number of different brain disorders including dementia $5,47-49$, epilepsy ${ }^{50-52}$, movement disorders ${ }^{53-55}$ and stroke 56,57 . Functional imaging is generally used in conjunction with structural neuroimaging and it is apparent that the accuracy and reliability of imaging used in research studies and in the clinic is improved by the application of increasingly sophisticated post-processing. For tests involving serial scanning of the same patient (such as epilepsy and stroke) it is important that the optimal acquisition and reconstruction are used for all scans and that the co-registration and scaling of scans is carried out accurately.

A number of different multi-centre studies have used FDG PET and DaTscan collected across different sites. This includes the Alzheimer's Disease Neuroimaging 
Initiative (ADNI) and the Parkinson's Progression Marker Initiative (PPMI) studies, both of which have used fillable phantoms to minimise inter-scanner differences ${ }^{44,58}$. Further validation of multi-centre studies could be gained by quantifying interscanner variation of the detectability of simulated disease states. The elliptical SSM phantom has been used to investigate differences in detectability of cold lesions in simulated HMPAO SPECT scans between gamma cameras ${ }^{59,60}$. It has also been applied to PET, to simulate higher activity tumour uptake ${ }^{17}$. The 3D printed anthropomorphic SSM phantom developed in this work has the potential to improve characterisation of intra and inter-scanner variation in these applications. 


\section{Conclusions}

It is important to quantify how accurately SPECT or PET scanners image the distribution of radionuclide within a patient, particularly when techniques are used to compare with previous images of that patient or with images of other patients or controls. Correction of images based on the characterisation of scanner systems will minimise variability between scanners and reconstruction software, and potentially enable detection of more subtle disease indications through existing and new techniques. Studies of the differing imaging characteristics of SPECT and PET scanners are limited due to difficulty in extracting reliable findings from the comparison of inherently variable human scanning, the lack of applicability of software simulation and the lack of realism of existing physical phantoms.

By using fused deposition modelling (3D printing) we have overcome a major limitation of existing emission tomography head phantoms. The tissue equivalence and anthropomorphic shape, derived from an MRI DICOM data set, enables automated attenuation correction routines to be applied to phantom scans. Improvements to the utility of the phantom would come from the addition of a skull and from thinner slabs to enable characterisation of PET imaging. Because the SSM phantom technique enables finely variable concentrations of radionuclide activity through grayscale printing on paper, it is more adaptable and more easily representative of complex clinical scans than fillable or cast phantoms. It is therefore suitable for clinically realistic comparisons of scanner response and for validation of numerical techniques to compare scans with normal databases for both SPECT and PET. 


\section{References}

1 J.M. Yeo, X. Lim, Z. Khan, and S. Pal, "Systematic review of the diagnostic utility of SPECT imaging in dementia.," Eur. Arch. Psychiatry Clin. Neurosci. 263(7), 539-52 (2013).

K.J. Van Laere, J. Warwick, J. Versijpt, I. Goethals, K. Audenaert, B. Van Heerden, and R. Dierckx, "Analysis of clinical brain SPECT data based on anatomic standardization and reference to normal data: an ROC-based comparison of visual, semiquantitative, and voxel-based methods," J Nucl Med 43(4), 458-469 (2002).

C. Huang, L.-O. Wahlund, O. Almkvist, D. Elehu, L. Svensson, T. Jonsson, B. Winblad, and P. Julin, "Voxel- and VOI-based analysis of SPECT CBF in relation to clinical and psychological heterogeneity of mild cognitive impairment," Neuroimage 19(3), 1137-1144 (2003).

H. Matsuda, S. Mizumura, T. Nagao, T. Ota, T. lizuka, K. Nemoto, M. Kimura, A. Tateno, A. Ishiwata, I. Kuji, H. Arai, and A. Homma, "An easy Z-score imaging system for discrimination between very early Alzheimer's disease and controls using brain perfusion SPECT in a multicentre study," Nucl Med Commun 28(3), 199-205 (2007).

M. Basely, M. Ceccaldi, L. Boyer, O. Mundler, and E. Guedj, "Distinct patterns of medial temporal impairment in degenerative dementia: a brain SPECT perfusion study in Alzheimer's disease and frontotemporal dementia," Eur J Nucl Med Mol Imaging 40(6), 932-942 (2013).

E. Imabayashi, H. Matsuda, T. Asada, T. Ohnishi, S. Sakamoto, S. Nakano, and T. Inoue, "Superiority of 3-dimensional stereotactic surface projection analysis over visual inspection in discrimination of patients with very early Alzheimer's disease from controls using brain perfusion SPECT," J Nucl Med 45(9), 1450-1457 (2004).

H. Matsuda, S. Mizumura, T. Nagao, T. Ota, T. lizuka, K. Nemoto, M. Kimura, A. Tateno, A. Ishiwata, I. Kuji, H. Arai, and A. Homma, "An easy Z-score imaging system for discrimination between very early Alzheimer's disease and 
controls using brain perfusion SPECT in a multicentre study.," Nucl. Med. Commun. 28(3), 199-205 (2007).

K.J. Van Laere, J. Versijpt, M. Koole, S. Vandenberghe, P. Lahorte, I.

Lemahieu, and R.A. Dierckx, "Experimental performance assessment of SPM for SPECT neuroactivation studies using a subresolution sandwich phantom design.," Neuroimage 16(1), 200-16 (2002).

L.R. Barnden, R.L. Hatton, S. Behin-Ain, B.F. Hutton, and E.A. Goble, "Optimisation of brain SPET and portability of normal databases," Eur J Nucl Med Mol Imaging 31(3), 378-387 (2004).

K. Van Laere, M. Koole, J. Versijpt, S. Vandenberghe, B. Brans, Y. D’Asseler, O. De Winter, A. Kalmar, and R. Dierckx, "Transfer of normal 99mTc-ECD brain SPET databases between different gamma cameras," Eur J Nucl Med 28(4), 435-449 (2001).

H. Matsuda, S. Mizumura, T. Soma, and N. Takemura, "Conversion of brain SPECT images between different collimators and reconstruction processes for analysis using statistical parametric mapping," Nucl Med Commun 25(1), 6774 (2004).

N.D. Smith, R.B. Holmes, M. Soleimani, M.J. Evans, S.C. Cade, and C.N. Mitchell, "Towards adapting a normal patient database for SPECT brain perfusion imaging," Inverse Probl. 28(6), (2012).

E.J. Hoffman, P.D. Cutler, W.M. Digby, and J.C. Mazziotta, "3-D phantom to simulate cerebral blood flow and metabolic images for PET," IEEE Trans. Nucl. Sci. 37(2), 616-620 (1990).

R. Wirestam, L. Andersson, L. Ostergaard, M. Bolling, J.P. Aunola, A. Lindgren, B. Geijer, S. Holtas, and F. Stahlberg, "Assessment of regional cerebral blood flow by dynamic susceptibility contrast MRI using different deconvolution techniques," Magn Reson Med 43(5), 691-700 (2000).

S.A. Larsson, C. Jonsson, M. Pagani, L. Johansson, and H. Jacobsson, "Original article A novel phantom design for emission tomography emission tomography imaging," Eur. J. Nucl. Med. 27(2), 131-139 (2000). 
HMPAO SPECT images using a subresolution sandwich phantom.," Neuroimage 81, 8-14 (2013).

B. Berthon, C. Marshall, R. Holmes, and E. Spezi, "A novel phantom technique for evaluating the performance of PET auto-segmentation methods in delineating heterogeneous and irregular lesions," EJNMMI Phys. 2(1), 13 (2015).

F. Rengier, A. Mehndiratta, H. von Tengg-Kobligk, C.M. Zechmann, R. Unterhinninghofen, H.-U. Kauczor, and F.L. Giesel, "3D printing based on imaging data: review of medical applications.," Int. J. Comput. Assist. Radiol. Surg. 5(4), 335-41 (2010).

H. lida, Y. Hori, K. Ishida, E. Imabayashi, H. Matsuda, M. Takahashi, H. Maruno, A. Yamamoto, K. Koshino, J. Enmi, S. Iguchi, T. Moriguchi, H. Kawashima, and T. Zeniya, "Three-dimensional brain phantom containing bone and grey matter structures with a realistic head contour.," Ann. Nucl. Med. 27(1), 25-36 (2013).

J.I. Gear, C. Long, D. Rushforth, S.J. Chittenden, C. Cummings, and G.D. Flux, "Development of patient-specific molecular imaging phantoms using a 3D printer," Med. Phys. 41(8), 082502 (2014).

M.A. Miller and G.D. Hutchins, "Development of Anatomically Realistic PET and PET / CT Phantoms with Rapid Prototyping Technology," in 2007 IEEE Nucl. Sci. Symp. Conf. Rec.(2007), pp. 4252-4257.

P.G. Hillel and D.L. Hastings, "The effect of phantom wall thickness on volume determination in SPET," Nucl. Med. Commun. 16, 1034-1040 (1995).

B. Berthon, C. Marshall, A. Edwards, M. Evans, and E. Spezi, "Influence of cold walls on PET image quantification and volume segmentation: A phantom study," Med. Phys. 40(8), 082505 (2013).

J.H. Hubbell and S. M. Seltzer, Tables of X-Ray Mass Attenuation Coefficients and Mass Energy-Absorption Coefficients from $1 \mathrm{keV}$ to $20 \mathrm{MeV}$ for Elements $Z=1$ to 92 and 48 Additional Substances of Dosimetric Interest, http://www.nist.gov/pml/data/xraycoef/index.cfm . Accessed : 28 Jan 2016. R. Bibb, D. Thompson, and J. Winder, "Computed tomography 
characterisation of additive manufacturing materials.," Med. Eng. Phys. 33(5), 590-6 (2011).

J. Solomon and E. Samei, "Quantum noise properties of CT images with anatomical textured backgrounds across reconstruction algorithms: FBP and SAFIRE.," Med. Phys. 41(9), 091908 (2014).

A.-K. Carton, P. Bakic, C. Ullberg, H. Derand, and A.D.A. Maidment, "Development of a physical 3D anthropomorphic breast phantom.," Med. Phys. 38(2), 891-6 (2011).

N. Kiarashi, A.C. Nolte, G.M. Sturgeon, W.P. Segars, S. V Ghate, L.W. Nolte, E. Samei, and J.Y. Lo, "Development of realistic physical breast phantoms matched to virtual breast phantoms based on human subject data.," Med. Phys. 42(7), 4116-26 (2015).

T.S. Yoo, T. Hamilton, D.E. Hurt, J. Caban, D. Liao, and D.T. Chen, "Toward quantitative X-ray CT phantoms of metastatic tumors using rapid prototyping technology," in 2011 IEEE Int. Symp. Biomed. Imaging From Nano to Macro(IEEE, 2011), pp. 1770-1773.

J. Madamesila, P. McGeachy, J.E. Villarreal Barajas, and R. Khan, "Characterizing 3D printing in the fabrication of variable density phantoms for quality assurance of radiotherapy," Phys. Medica (2015).

R. Jones, P. Haufe, E. Sells, P. Iravani, V. Olliver, C. Palmer, and A. Bowyer, "RepRap - the replicating rapid prototyper," Robotica 29(01), 177-191 (2011). pronterface, http://www.pronterface.com/ . Accessed : 28 Jan 2016. Slic3r, www.slic3r.org . Accessed : 28 Jan 2016.

A.A. Konstas, G. V Goldmakher, T.-Y. Lee, and M.H. Lev, "Theoretic basis and technical implementations of CT perfusion in acute ischemic stroke, part 2: technical implementations.," AJNR. Am. J. Neuroradiol. 30(5), 885-92 (2009). C.A. Cocosco, V. Kollokian, R.K.S. Kwan, and A.C. Evans, "BrainWeb: Online Interface to a 3D MRI Simulated Brain Database," in Neurolmage - Proc. 3rd Int. Conf. Funct. Mapp. Hum. Brain(1997), p. S425. 
and A.C. Evans, "Design and construction of a realistic digital brain phantom.," IEEE Trans. Med. Imaging 17(3), 463-8 (1998).

R. K. Kwan, A.C. Evans, and G.B. Pike, "An Extensible MRI Simulator for Post-Processing Evaluation," in Lect. Notes Comput. Sci. - Vis. Biomed. Comput.(Springer-Verlag, 1996), pp. 135-140.

R.K. Kwan, A.C. Evans, and G.B. Pike, "MRI simulation-based evaluation of image-processing and classification methods.," IEEE Trans. Med. Imaging 18(11), 1085-97 (1999).

P. Yushkevich, J. Piven, H.C. Hazlett, R.G. Smith, S. Ho, J.C. Gee, and G. Gerig, "User-guided 3D active contour segmentation of anatomical structures: significantly improved efficiency and reliability.," Neuroimage 31(3), 1116-28 (2006).

A. Fedorov, R. Beichel, J. Kalpathy-Cramer, J. Finet, J.-C. Fillion-Robin, S. Pujol, C. Bauer, D. Jennings, F. Fennessy, M. Sonka, J. Buatti, S. Aylward, J. V Miller, S. Pieper, and R. Kikinis, "3D Slicer as an image computing platform for the Quantitative Imaging Network.," Magn. Reson. Imaging 30(9), 1323-41 (2012).

S.R. Cherry, J.A. Sorenson, and M.E. Phelps, Physics in Nuclear Medicine, 4th ed. (Elsevier Saunders, Philadelphia, 2012).

R.B. Holmes, Analysis of Functional Neuroimaging (University of Bristol, 2011).

SPM, http://www.fil.ion.ucl.ac.uk/spm/ . Accessed : 19 May 2016.

A. Joshi, R.A. Koeppe, and J.A. Fessler, "Reducing between scanner differences in multi-center PET studies," Neuroimage 46(1), 154-159 (2009). H. lida, Y. Narita, H. Kado, A. Kashikura, S. Sugawara, Y. Shoji, T. Kinoshita, T. Ogawa, and S. Eberl, "Effects of scatter and attenuation correction on quantitative assessment of regional cerebral blood flow with SPECT.," J. Nucl. Med. 39(1), 181-189 (1998).

R.Z. Stodilka, B.J. Kemp, F.S. Prato, and R.L. Nicholson, "Importance of bone attenuation in brain SPECT quantification.," J. Nucl. Med. 39(1), 190-7 (1998). 
A. Swan, B. Waddell, G. Holloway, T. Bak, S. Colville, Z. Khan, and S. Pal, "The diagnostic utility of 99mTc-HMPAO SPECT imaging: a retrospective case series from a tertiary referral early-onset cognitive disorders clinic.," Dement. Geriatr. Cogn. Disord. 39(3-4), 186-93 (2015).

R.K.J. Brown, N.I. Bohnen, K.K. Wong, S. Minoshima, and K.A. Frey, "Brain PET in suspected dementia: patterns of altered FDG metabolism.," Radiographics 34(3), 684-701 (2014).

T. Yamane, Y. Ikari, T. Nishio, K. Ishii, T. Kato, K. Ito, D.H.S. Silverman, M. Senda, T. Asada, H. Arai, M. Sugishita, and T. Iwatsubo, "Visual-statistical interpretation of (18)F-FDG-PET images for characteristic Alzheimer patterns in a multicenter study: inter-rater concordance and relationship to automated quantitative evaluation.," AJNR. Am. J. Neuroradiol. 35(2), 244-9 (2014).

K. Jafari-Khouzani, K. Elisevich, K.C. Karvelis, and H. Soltanian-Zadeh, "Quantitative multi-compartmental SPECT image analysis for lateralization of temporal lobe epilepsy," Epilepsy Res 95(1-2), 35-50 (2011).

N.J. Kazemi, G.A. Worrell, S.M. Stead, B.H. Brinkmann, B.P. Mullan, T.J. O'Brien, and E.L. So, "Ictal SPECT statistical parametric mapping in temporal lobe epilepsy surgery," Neurology 74(1), 70-76 (2010).

D. Pustina, B. Avants, M. Sperling, R. Gorniak, X. He, G. Doucet, P. Barnett, S. Mintzer, A. Sharan, and J. Tracy, "Predicting the laterality of temporal lobe epilepsy from PET, MRI, and DTI: A multimodal study," Neurolmage Clin. 9, 20-31 (2015).

M. Sudmeyer, C. Antke, T. Zizek, M. Beu, S. Nikolaus, L. Wojtecki, A. Schnitzler, and H.-W. Muller, "Diagnostic Accuracy of Combined FP-CIT, IBZM, and MIBG Scintigraphy in the Differential Diagnosis of Degenerative Parkinsonism: A Multidimensional Statistical Approach," J. Nucl. Med. 52(5), 733-740 (2011).

K. Van Laere, C. Casteels, L. De Ceuninck, B. Vanbilloen, A. Maes, L. Mortelmans, W. Vandenberghe, A. Verbruggen, and R. Dom, "Dual-tracer dopamine transporter and perfusion SPECT in differential diagnosis of parkinsonism using template-based discriminant analysis," J Nucl Med 47(3), 384-392 (2006). 
S. Thobois, M. Jahanshahi, S. Pinto, R. Frackowiak, and P. Limousin-Dowsey, "PET and SPECT functional imaging studies in Parkinsonian syndromes: from the lesion to its consequences," Neuroimage 23(1), 1-16 (2004).

J.T. Whisenant, Y. Kadkhodayan, D.T. Cross, C.J. Moran, and C.P. Derdeyn, "Incidence and mechanisms of stroke after permanent carotid artery occlusion following temporary occlusion testing.," J. Neurointerv. Surg. (2014).

M. Yamauchi, E. Imabayashi, H. Matsuda, J. Nakagawara, M. Takahashi, E. Shimosegawa, J. Hatazawa, M. Suzuki, H. Iwanaga, K. Fukuda, K. lihara, and H. lida, "Quantitative assessment of rest and acetazolamide CBF using quantitative SPECT reconstruction and sequential administration of (123)Iiodoamphetamine: comparison among data acquired at three institutions.," Ann. Nucl. Med. 28(9), 836-50 (2014).

K. Marek, D. Jennings, S. Lasch, A. Siderowf, C. Tanner, T. Simuni, C. Coffey, K. Kieburtz, E. Flagg, S. Chowdhury, W. Poewe, B. Mollenhauer, T. Sherer, M. Frasier, C. Meunier, A. Rudolph, C. Casaceli, J. Seibyl, S. Mendick, N. Schuff, Y. Zhang, A. Toga, K. Crawford, A. Ansbach, and P. Taylor, "The Parkinson Progression Marker Initiative (PPMI).," Prog. Neurobiol. 95(4), 629-35 (2011). R.B. Holmes, D. Martin, L. Tossici-Bolt, L. Jenkins, N. Bush, and P.M. Kemp, "Multi-Camera Comparison of HMPAO SPECT Simulated Lesion Detectability Using a Subresolution Sandwich Phantom.," Eur. J. Nucl. Med. Mol. Imaging 35(Supp 2), S176; 298 (2008).

R.B. Holmes, M.J. Evans, N.D. Smith, and P.M. Kemp, "A Study of HMPAO SPECT Database Portability Using a Subresolution Sandwich Phantom," Eur. J. Nucl. Med. Mol. Imaging 38(Supp 2), S174; OP392 (2011).

\section{Conflicts of Interest}

None

\section{Acknowledgements}

$\mathrm{R} \mathrm{H}$ acknowledges the support of a UK National Institute for Health Research post- 
doctoral research fellowship. DN carried out work as part of an MSc thesis for the University of Surrey, UK. KJ carried out work as part of a BSc thesis for the University of Bristol, UK. 